\title{
CORRECTION
}

Check for updates

Cite this: J. Mater. Chem. A, 2018, 6, 1865

DOI: $10.1039 / c 7 t a 90287 e$

www.rsc.org/MaterialsA

\section{Correction: MXene: an emerging two-dimensional material for future energy conversion and storage applications}

\author{
Nitin K. Chaudhari, ${ }^{\text {ab }}$ Hanuel Jin, ${ }^{a}$ Byeongyoon Kim, ${ }^{a}$ Du San Baek, ${ }^{c}$ Sang Hoon Joo*c \\ and Kwangyeol Lee*a \\ Correction for 'MXene: an emerging two-dimensional material for future energy conversion and storage \\ applications' by Nitin K. Chaudhari et al., J. Mater. Chem. A, 2017, 5, 24564-24579.
}

The authors regret an omission in the acknowledgement of a referenced study.

Ref. 25 in the original manuscript (M. Naguib, M. Kurtoglu, V. Presser, J. Lu, J. Niu, M. Heon, L. Hultman, Y. Gogotsi and M. W. Barsoum, Adv. Mater., 2011, 23, 4248) was performed jointly by the Y. Gogotsi and M. W. Barsoum groups at Drexel University. In order to give proper credit to both groups, the authors would like to change the original sentence "A simple but very effective approach to extract the A layers from the MAX phase, involving HF treatment followed by ultrasonication to facilitate the exfoliation into 2D MXenes, was first reported by Gogotsi's group. ${ }^{25}$ " to "A simple but very effective approach to extract the A layers from the MAX phase, involving HF treatment followed by ultrasonication to facilitate the exfoliation into 2D MXenes, was first reported jointly by Barsoum and Gogotsi groups. ${ }^{25}$,

The Royal Society of Chemistry apologises for these errors and any consequent inconvenience to authors and readers. 